\title{
ALCUNE RIFLESSIONI CONTRASTIVE SUL VERBO
}

\section{Introduzione}

Scopo del presente lavoro è di offrire un modesto contributo alla formazione del lessico italiano. L'articolo tratta la formazione suffissale dei verbi sia in croato che in italiano. Prende in considerazione un gruppo di parole formate: è un gruppo di verbi derivati mediante suffissi, un gruppo non molto numeroso, ma interessante dal punto di vista formativo e soprattutto dal punto di vista semantico. Si esaminano i suffissati verbali, formati da basi che sono voci onomatopeiche o di origine onomatopeica, interiezioni o esclamazioni o anche altre parole monosillabe e bisillabe. Abbiamo esaminato i verbi croati e i verbi italiani, abbiamo cioè esaminato gli equivalenti formali e semantici dei verbi croati nella lingua italiana. Confrontando questi verbi abbiamo cercato di mettere in rilievo le caratteristiche formali e quelle semantiche dei verbi, nonché di sottolineare i problemi che si possono incontrare nella traduzione dei rispettivi verbi. Affrontando questo tema, pur sempre rimanendo nell'ambito della formazione delle parole, abbiamo cercato di ampliare un po' il tema e in particolare abbiamo voluto fissare un punto di avvio per ulteriori ricerche e analisi.

\section{I verbi e i loro equivalenti}

2.1. I verbi croati onomatopeici blejati bleketati, meketati e veketati si riferiscono agli animali e in particolare alle pecore, agli agnelli e alle capre. L'equivalente formale e semantico in italiano è il verbo belare 'emettere belati'. ' Il verbo belare e l'interiezione "bè" o "bèe" riproducono bene il verso lamentoso caratteristico delle pecore, degli agnelli e delle capre. Usato in senso figurato il verbo esprime il frignare e piagnucolare lamentoso, parlando soprattutto dei bambini; il parlare noioso, inutile e molesto; e la recitazione lamentosa o il canto lamentoso: belare una poesia, una canzone.

1 Il verbo italiano belare continua il latino tardo bēlāre per il classico bālāre, bālātus; la voce latina è di origine onomatopeica; deriva dall'interiezione "bee" che riproduce il belato delle pecore, degli agnelli e delle capre. 
2.2. Il verbo croato blebetati esprime il conversare futile, il parlare del più e del meno. Il suo equivalente semantico in italiano è il verbo onomatopeico chiacchierare 'conversare futilmente'. Le voci onomatopeiche "bla bla" o "blablà" o "blablablà" esprimono quindi lo stesso concetto: 'chiacchiericcio futile, discorso inutile e senza costrutto, senza conclusione'.

2.3. Il verbo croato onomatopeico cijukati è formato dalla voce onomatopeica "ciju"; può riprodurre il lamento del cane ed allora il suo equivalente semantico è il verbo guaire, formato dall'esclamazione "guai" che esprime dolore e riproduce quindi l'abbaiare lamentoso del cane che guaisce per il dolore o emette guaiti. Da guaire è stato formato il verbo guaiolare, sul tipo iterativo di miagolare o gnaulare. Usato in senso figurato e con valore spregiativo il verbo onomatopeico guaire può esprimere il lamentarsi detto di persone, e in particolare il parlare o il cantare con toni lamentosi e striduli'.

2.3.1. Il verbo croato cijukati riproduce anche il verso caratteristico dei pulcini o degli uccellini da nido e allora il suo equivalente italiano è il verbo pigolare di origine onomatopeica 'emettere pigolii' detto degli uccelletti o uccellini da nido e dei pulcini che pigolano. ${ }^{2}$ II verbo pigolare viene usato anche in senso figurato e allora significa 'lamentarsi, piagnucolare in modo insistente'.

2.3.2. Il verbo cijukati esprime pure il verso stridulo di alcuni animali, e in particolare di animali giovani, del papagallo e del topo e in questo uso il suo equivalente italiano è il verbo squittire.

2.4. Il verbo croato cvrčati esprime il verso caratteristico della cicala: lo stridere delle cicale. Il suo equivalente italiano frinire è di origine onomatopeica. ${ }^{3}$ Riproduce il verso stridulo e acuto della cicala che frinisce, ma anche stride. Il suono meno acuto, un canto lieve e ripetuto di molti altri uccelli, e specialmente di passeri, lo si può esprimere con un altro verbo onomatopeico cinguettare 'cvrkutati' o con il verbo gorgheggiare 'cvrkutati', che non è di origine onomatopeica: l'usignolo e il canarino gorgheggiano. Il verbo cvrčati significa anche 'emettere scoppietti, crepitii caratteristici di cosa che frigge' e nella traduzione si usano i verbi sfriggere o sfrigolare: la carne sfrigge nell'olio bollente. Riproduce anche il crepitio del legno verde che arde. Usato in senso figurato il verbo gorgheggiare si riferisce a persone: il cantante gorgheggia. $\mathrm{E}$ il verbo cinguettare, usato in senso figurato, si riferisce a bambini o a chi imita il linguaggio dei bambini 'parlare balbettando', detto di bambini e di chi bambineggia.

2.5. Il verbo croato onomatopeico ćurlikati esprime il canto allegro, il cinguettìo o il gorgheggio degli uccelli e in particolare dell'allodola e il canto ripetuto della quaglia. Gli equivalenti italiani sono il verbo trillare, che deriva da trillo di origine onomatopeica, 'emettere trilli' detto dell'allodola che emette durante il volo un trillo armonioso, o i verbi gorgheggiare e cinguettare.

3 Il verbo italiano frinire è una voce dotta e continua il latino frītinnīre onomatopeico. 
2.6. Il verbo croato gakati è formato dall' interiezione onomatopeica "ga" che riproduce il verso caratteristico delle oche e delle cornacchie. Esiste anche il verbo graktati. Si traducono con i verbi italiani gracchiare e schiamazzare. ${ }^{4}$ Il verbo gracchiare riproduce il cantare rauco, caratteristico di alcuni uccelli, e specialmente di corvi e cornacchie; e il verbo schiamazzare riproduce il grido rauco e scomposto di volatili e in particolare di oche. Usati in senso figurato questi verbi si riferiscono a persone: gracchiare 'ciarlare o brontolare fastidiosamente' (smettila di gracchiare!) e schiamazzare 'vociare, strepitare' detto di persone.

2.7. I verbi croati onomatopeici glogotati, grgoljiti, grgotati, grgoriti e klokotati riproducono il rumoreggiare dell'acqua che scorre tra i sassi, dell'acqua della fontana, delle onde e dell'acqua che scaturisce o bolle. L'equivalente italiano è il verbo onomatopeico gorgogliare.

2.7.1. Il verbo italiano gloglottare, formato dall'interiezione onomatopeica "glo glo" (usata nelle locuzioni verbali 'fare glo glo o fare glu glu'), è una voce onomatopeica che riproduce il verso caratteristico del tacchino e della faraona. Usato in senso figurato ha tutti i significati del verbo gorgogliare ed esprime il rumoreggiare dell' acqua: l'acqua gloglotta scorrendo, il ruscello gloglotta.

2.8. Il verbo gukati è formato dall'interiezione onomatopeica "gu". L'equivalente italiano è il verbo onomatopeico tubare $e^{5}$ che esprime il gridare di colombi, di tortore: è un caratteristico grido gutturale che essi emettono specialmente durante il periodo dell'accoppiamento. E l'interiezione onomatopeica "tubi tubi" è voce di richiamo per i piccioni. Il verbo italiano tubare può essere usato anche in senso figurato 'amoreggiare teneramente': tubano come due colombi.

2.9. I verbi croati groktati e roktati sono verbi onomatopeici. I loro equivalenti italiani sono grugnire e grugnare che riproducono il verso caratteristico del maiale che grugnisce. ${ }^{6}$ Il verbo grugnire, usato in senso figurato, si riferisce a persone: 'parlare in modo sgradevole e sconveniente'. Il verbo onomatopeico grugnire, oltre a essere intransitivo, può essere anche transitivo e allora regge un oggetto diretto; in questo caso si riferisce pure a persone 'dire q.c.in modo poco chiaro': grugnire un saluto.

2.10. Il verbo croato hukati, e i verbi croati hučati e hujati, sono verbi onomatopeici. Riproducono il verso animale. Il loro equivalente formale e semantico in italiano è il verbo ululare di origine onomatopeica 'il gridare lungo e lamentoso di lupi, cani ecc.', 'urlare'. 7

4 Il verbo italiano gracchiare è un denominale da gracchio 'un tipo di cornacchia', proviene dal latino gräculus che è una voce onomatopeica che riproduce il verso caratteristico e abituale del gracchio. Il verbo italiano schiamazzare proviene dal verbo latino exclamāre (ex-clamāre) 'chiamare ad alta voce'.

5 Il verbo tubare continua il latino tardo tutubāre 'gridare della civetta' di origine onomatopeica: dal verso "tu tu" di questo uccello.

6 I verbi italiani grugnire e grugnare di origine onomatopeica continuano il latino grunnïre (grundire), formato con sovrapposizione del parallelo verbo del latino tardo grunniāre; la base onomatopeica di tutti e due i verbi latini è l'interiezione onomatopeica *"gru" detto del porco. 
2.10.1. Il verbo ululare, usato in senso figurato, riproduce il suono del vento, della bufera, dell'uragano, della tempesta, del mare. Lo stesso rumore del vento o dell'acqua può essere espresso anche dai verbi fischiare e sibilare (si traducono in croato anche con fijukati): il vento fischia tra i rami, il vento ulula nella valle, il vento sibila tra le vele, la bufera ulula nella selva. Usati in senso figurato anche i verbi fremere, mugghiare e muggire esprimono il rumoreggiare cupo del vento, della bufera, della tempesta, dell'uragano, della cascata, del torrente, del mare e del tuono.

2.11. I verbi croati kevtati e štektati sono verbi onomatopeici che riproducono il verso caratteristico degli animali, e in particolare il latrare del cane, l'abbaiare ripetuto, intenso e prolungato del cane durante la caccia alla selvaggina; gli stessi verbi possono riprodurre anche il verso del lupo e della tigre. Riproducono anche il mugolìo degli animali, e in particolare il mugolìo del cane, e cioè riproducono suoni continui e frequenti del cane, suoni indistinti e lamentosi che esso produce tenendo la bocca chiusa.

2.11.1. L'equivalente italiano è il verbo mugolare. $^{8}$ Usato in senso figurato, si riferisce a persone ed esprime il lamento, il gemito: mugolare di dolore, di piacere. Il verbo italiano mugolare, oltre ad essere intransitivo, può essere anche transitivo e allora regge un oggetto diretto; anche in questo caso si riferisce a persone ed esprime il mormorare e il borbottare: mugolare tra sé strane parole.

2.12. Il verbo croato kmečati è un derivato di origine onomatopeica, formato dall'interiezione onomatopeica "kme" che riproduce il vagito dei bambini lattanti. Il suo equivalente semantico in italiano è il verbo vagire ${ }^{9}$ che esprime il pianto del bambino piccolo.

2.13. I verbi croati kokodakati e kokotati sono verbi onomatopeici formati dal segno convenzionale "ko-ko". L'equivalente italiano è la locuzione verbale analitica e onomatopeica far coccodè che riproduce il verso della gallina quando ha fatto l'uovo. Si impiega pure come nome di genere maschile: Il pollaio risuona di coccodè.

2.13.1. Anche il verbo italiano schiamazzare può riprodurre gridi rauchi e scomposti di animali: delle galline e di altri volatili, specialmente delle oche. Quando viene usato in senso figurato, il verbo schiamazzare esprime il vociare e lo strepitare delle persone.

2.14. Il verbo croato kreketati è formato dall'esclamazione onomatopeica "kre-kre", verso caratteristico delle ranocchie. L'equivalente italiano è il verbo gracidare di origine onomatopeica che riproduce il gracidare delle rane. ${ }^{10} \mathrm{Il}$ verbo

7 Il verbo italiano onomatopeico ululare è una voce dotta; il verbo deriva dal latino ululāre di origine onomatopeica, derivato dall'ulula 'allocco'; esprime il verso dell'ulula, anche voce dotta di origine onomatopeica.

8 Il verbo italiano mugolare è una voce semidotta, continua il latino tardo *mūgulāre, sviluppatosi dal latino $m \bar{u} g \bar{i} r e$, di origine onomatopeica.

9 Il verbo vagire è voce dotta, proviene dal latino vāgīre, vāgītus di origine onomatopeica.

10 Il verbo italiano gracidare continua il latino tardo grācitāre, che è un verbo onomatopeico ed 
gracidare può essere impiegato anche in senso figurato 'parlare in modo noioso, con voce lamentosa e stridula'.

2.15. Il verbo croato kucati è formato dalla base onomatopeica che si scrive convenzionalmente "kuc" o "kuc-kuc" e che riproduce il picchiare alla porta, leggero e ripetuto, con il dito, con il martello e sim.; l'equivalente italiano è il verbo picchiare, anche di origine onomatopeica 'colpire o battere ripetutamente'.

2.16. La base del verbo croato kukati è l'interiezione onomatopeica "kuku" con cui si designá convenzionalmente il verso del cuculo; l'equivalente formale e semantico in italiano è il verbo cuculiare, formato dall'interiezione onomatopeica "cucù" o "cuccù" o "cu cu"; il verbo è abbastanza raro e si preferisce impiegare l'espressione fare il verso del cuculo. In senso figurato, nei giochi dei bambini, si impiega la locuzione verbale fare cu cu 'far capolino' e l' interiezione "cucù" o "cuccù" o "cu cu" come richiamo fra i bambini che giocano a nascondersi.

2.17. I verbi onomatopeici kukurikati e kukurijekati sono formati dall'interiezione onomatopeica "kukuriku" con cui si designa convenzionalmente il verso del gallo. L'equivanente formale e semantico in italiano è il verbo onomatopeico chicchiriare, formato dalla voce onomatopeica accorciata "chicchirichì". La locuzione verbale analitica far chicchirichì riproduce ancora meglio il canto del gallo che emette il caratteristico canto acuto, sonoro e prolungato.

2.18. I verbi onomatopeici maukati, mijaukati, mjaukati, formati da voci onomatopeiche "mijau" o "mjau", hanno almeno due equivalenti formali e semantici: miagolare e gnaulare, tutti e due verbi onomatopeici; il verbo miagolare, formato dalla voce onomatopeica "miao" o "miau", quindi 'fare miao, miao' detto del gatto e il verbo gnaulare, formato dalla voce onomatopeica "gnao" o "gnau", quindi 'fare gnao o gnau'. Possono essere usati anche in senso figurato: gnaulare 'lamentarsi fastidiosamente' e miagolare 'lamentarsi', detto scherzosamente: piantala di miagolare in quel modo!

2.19. Il verbo croato onomatopeico mukati 'dire mu' detto dei buoi ha almeno due equivalenti formali e semantici di origine onomatopeica in italiano: muggire 'emettere muggiti' detto dei buoi e mugghiare 'muggire forte e lungamente' anche detto dei buoi. ${ }^{11}$

2.20. I verbi nanati, ninati e nunati sono verbi onomatopeici. L'equivalente italiano è il verbo ninnare, formato dalla voce infantile ninna 'il sonno, il dormire': ninnare 'cullare', cantare la ninnananna', conciliare il sonno'. Le voci infantili nanna e ninna formano con il verbo fare le locuzioni verbali: fare la nanna e fare la ninna 'addormentarsi', dormire'. E la voce infantile nanna forma con altri verbi neutri locuzioni verbali come andare a nanna, mettere a nanna.

esprime il verso caratteristico dei ranocchi.

11 Il verbo italiano muggire 'mugghiare' continua il latino mūgīre, mūgītus; il verbo mugghiare proviene dal verbo del latino tardo *mūgulāre per il classico mūgilāre. Gli usi figurati dei verbi italiani muggire e mugghiare sono stati già trattati come equivalenti semantici del verbo hukati. 
2.21. Il verbo croato njakati, 'pronunciare nja', è un verbo onomatopeico formato dalla base "nja", che è un'interiezione onomatopeica. Il verbo sinonimo è revati. L'equivalente italiano è il verbo ragliare ${ }^{12}$ che riproduce il gridare dell'asino': 'mandare o emettere uno o più ragli', detto dell'asino che raglia. Usato in senso figurato il verbo ragliare riproduce il rumoreggiare cupo del tuono, del mare, del vento.

2.21.1. Può essere riferito a persone e allora significa 'gridare come un asino', e poi 'parlare, cantare sgradevolmente o dire sciocchezze': non fa che ragliare.

2.22. Il verbo njiskati, 'pronunciare njis', e il verbo njištati sono verbi onomatopeici. Il verbo sinonimo è rzati. Il loro equivalente formale e semantico in italiano è il verbo nitrire. ${ }^{13}$ Riproduce il grido penetrante del cavallo, il verso caratteristico del cavallo che nitrisce.

2.23. Il verbo pijukati di origine onomatopeica è formato dall'esclamazione onomatopeica "piju-piju", con cui si imita il verso dei pulcini che camminano dietro la chioccia e si usa come richiamo quando si distribuisce il mangime ai pulcini o quando si vuole radunarli. Il suo equivalente formale e semantico in italiano è il verbo pigolare,$^{14}$ anche voce onomatopeica 'il bisbigliare dei pulcini e in particoalre degli uccelli giovani'. Il verbo riproduce bene il verso breve, sommesso e ripetuto di piccoli animali pennuti: gli uccelletti ed i pulcini pigolano.

23.1. E all'esclamazione croata "piju-piju" equivale in italiano l'esclamazione onomatopeica "pio pio" o "pi pi". Usato in senso figurato il verbo pigolare significa 'lamentarsi', piagnucolare in modo insistente: smettila di pigolare in quel modo!

2.24. Il verbo croato zujati è un verbo onomatopeico formato dall'interiezione onomatopeica che si scrive convenzionalmente come "zzz" e riproduce il ronzio di un insetto, il caratteristico rumore sordo e vibrante detto di zanzare, vespe, mosconi, api e sim. o semplicemente il volare detto di un insetto. L'equivalente semantico è il verbo ronzare, anche voce onomatopeica. Usato in senso figurato il verbo ronzare significa 'girare, mulinare': molte idee gli ronzano in testa; uno può ronzare intorno a un luogo o una persona; o uno può ronzare intorno a una ragazza per corteggiarla.

\section{Conclusione}

1. Prendendo in esame solo un segmento di formazione dei verbi, abbiamo cercato di sottolineare alcune caratteristiche di questo gruppo di verbi nell'ambito della formazione delle parole, caratteristiche sia formali che semantiche, al fine di accennare a problemi che si incontrano nella traduzione.

12 Il verbo italiano ragliare continua il latino tardo * ragulāre di origine onomatopeica.

13 Il verbo italiano nitrire continua il verbo latino hinnīre, che deriva dal nome latino hinnītus 'nitrito', di origine onomatopeica, attraverso un tardo latino hinnitīre.

14 Il verbo italiano pigolare continua il latino tardo * piulalare di origine onomatopeica. 
2. I verbi presi in considerazione nel presente articolo sono verbi onomatopeici o di origine onomatopeica. Questi verbi sono formati da voci onomatopeiche, da interiezioni o da altre parole monosillabe o polisillabe; tutti sono formati mediante suffissi.

3. I suffissi croati che servono per formare questi verbi sono -ati, -čati, -etati, -jati, -kati, -ketati, -otati, -tati. E gli equivalenti italiani sono formati mediante i suffissi -are, -azzare, -eggiare, -ere, -ire.

4. Questi verbi onomatopeici o di origine onomatopeica, nonché i verbi formati da interiezioni o da altre parole monosillabe o polisillabe riproducono prevalentemente $i l$ verso caratteristico degli animali - animali domestici, animali selvatici, uccelli.

4.1. Però, gli stessi verbi, usati in senso figurato, esprimono suoni o nozioni appartenenti al linguaggio umano, alla maniera di parlare umana.

4.2. Spesso essi riproducono il suono del vento - il suono della bufera, della tempesta, dell'uragano; il suono dell'acqua - il suono della sorgente, della cascata, del torrente, del mare, delle onde ed il suono del tuono.

4.3. In alcuni casi $\mathrm{i}$ verbi designano suoni o nozioni appartenenti al mondo infantile.

\section{Bibliografia}

Anić, V. (1994) Rječnik hrvatskoga jezika, Novi Liber, Zagreb

Babić, S. (1991) Tvorba riječi u hrvatskom književnom jeziku. Nacrt za gramatiku.

Hrvatska akademija znanosti i umjetnosti, Globus, Zagreb.

Battaglia, S. (1961-1994) Grande dizionario della lingua italiana, UTET, Torino.

Battisti, C.-Alessio, G. (1966) Dizionario etimologico italiano, I-V, Barbèra, Firenze.

Cortelazzo, M.-Zolli, P. (1979) Dizionario etimologico della lingua italiana, Bologna.

Dardano, M. (1978) La formazione delle parole nell'italiano di oggi, Bulzoni, Roma.

Dardano, M. (1981) Preliminari per lo studio della formazione delle parole nell'italiano di oggi. Aspetti lessicologici e semantici, in "SLI: Lessico e semantica", Bulzoni, Roma, pp. 275-291.

Deanović, M.-Jernej, J. (1992) Talijansko-hrvatski rječnik, Školska knjiga, Zagreb.

Deanović, M.-Jernej, J. (1991) Hrvatsko-talijanski rječnik, Školska knjiga, Zagreb.

Devoto, G. (1968) Avviamento all'etimologia italiana, Le Monnier, Firenze.

Devoto, G.-Oli, G.C. (1987) Il Nuovo Vocabolario Illustrato della Lingua Italiana, Se-

lezione dal Reader's Digest, Milano.

Garzanti ed., (1987) Il Grande Dizionario Garzanti della lingua italiana, Milano.

Regula, M.-Jernej, J. (1975) Grammatica italiana descrittiva su basi storiche e psicologiche, Franche Verlagh, Bern.

Tekavčić, P. (1980) Grammatica storica dell' italiano, III: Lessico, il Mulino, Bologna.

Vučetić, Z. (1979) Suvremeni talijanski jezik-Tvorba riječi, Liber, Zagreb. 
Vučetić, Z. (1996) Contributo allo studio della suffissazione verbale nell'italiano contemporaneo. Raffronto contrastivo, Linguistica XXXVI, Ljubljana, pp. 83-96.

Zingarelli, N. (1996) Vocabolario della lingua italiana, Dodicesima edizione, Zanichelli, Bologna.

\section{Povzetek \\ KONTRASTIVNA RAZMIŠLJANJA O GLAGOLU}

Prispevek ostaja v okviru besedne tvorbe, natančneje tvorbe glagola s pomočjo pripon. Primerjamo glagol v italijanskem in hrvatskem jeziku, tako glede na obliko kot na pomen. Opozarjamo na probleme pri prevajanju takih glagolov.

$\mathrm{V}$ prispevku tehtamo glagole, izpeljane s priponami, in sicer tiste glagole, za katere lahko predpostavimo onomatopejski, posnemovalni vir. Največkrat je to posnemovalni medmet. V hrvaškem jeziku so pripone, ki se pritikajo, -ati, -čati, -etati, -jati, -kati, -ketati, -otati, -tati. Njihove ustreznice v italijanščini pa so glagoli, tvorjeni s priponami -eggiare, -azzare, včasih pa taka izpeljava iz glagolskega morfema ni vidna (-are, -ere, -ire).

Ponajvečkrat služijo ti glagoli za izražanje značilnih glasov živali, tako domačih kot divjih, pa tudi glasov ptic. Rabljeni v prenesenem pomenu so lahko del človeškega govora, dokaj redko jih najdemo v otroškem govoru. Včasih se jih človek posluži za posnemanje zvokov v neživi naravi: vetra, viharja, vode, izvirkov, slapa, morja, valov, groma. 